\title{
CONTINUATION OF BIFURCATIONS IN PERIODIC DELAY-DIFFERENTIAL EQUATIONS USING CHARACTERISTIC MATRICES
}

\author{
RÓBERT SZALAI*, GÁBOR STÉPÁN ${ }^{\dagger}$, AND S. JOHN HOGAN ${ }^{\ddagger}$
}

\begin{abstract}
In this paper we describe a method for continuing periodic solution bifurcations in periodic delay-differential equations. First, the notion of characteristic matrices of periodic orbits is introduced and equivalence with the monodromy operator is demonstrated. An alternative formulation of the characteristic matrix is given, which can be computed efficiently. Defining systems of bifurcations are constructed in a standard way including the characteristic matrix and its derivatives. For following bifurcation curves in two parameters, the pseudo-arclength method is used combined with Newton iteration. Two test examples (an interrupted machining model and a traffic model with driver reaction time) conclude the paper. The algorithm has been implemented in the software tool, PDDE-CONT.
\end{abstract}

Key words. delay-differential equations, periodic solutions, bifurcations, continuation

AMS subject classifications. 37M20, 34K13, 34K18

1. Introduction. Continuation methods have wide-spread use in scientific applications described by ordinary differential equations (see e.g. $[6,9,21]$ ). These methods are used to investigate invariant structures, such as fixed points and periodic orbits of dynamical systems over a range of parameter values. Usually only special or trivial solutions are available analytically and direct simulations only converge to stable (or unstable, if time reversed) solutions. Numerical continuation methods provide an ideal tool to find bifurcations of these solutions even after they become of saddle type. Similarly, bifurcations can also be continued by allowing additional parameters to vary.

However when delay differential equations (DDEs) $[7,17,18]$ are considered, the resulting infinite dimensional system presents far greater numerical challenges. Available continuation methods for bifurcations in DDEs are essentially limited to fixed points. The software DDE-BIFTOoL, developed by Engelborghs et al. [13], is capable of continuing bifurcations of fixed points. It can also continue periodic orbits, but not their bifurcations. To compute fixed point bifurcations, Engelborghs et al. [13] used the characteristic matrix [17], which can be computed analytically when the partial derivatives of the vector field are known. They exploit the fact that this matrix is singular at bifurcation points. In this paper we use a similar approach, but for periodic orbits.

The paper is organized as follows. In Section 2, we introduce the characteristic matrix for periodic orbits. We derive its equivalence with the monodromy operator and show how its computational cost can be reduced to that of the solution of one system of linear equations. In Section 3, continuation of periodic orbits is discussed using the pseudo-arclength method. Continuation of bifurcations is discussed in Section 4. In Section 5, we give implementation details of the algorithm. In Section 6, two examples, an interrupted machining model and a traffic model, are analyzed.

\footnotetext{
*Budapest University of Technology and Economics, Department of Applied Mechanics, H-1521, Budapest, Hungary szalai@mm.bme.hu

${ }^{\dagger}$ Budapest University of Technology and Economics, Department of Applied Mechanics, H-1521, Budapest, Hungary stepan@mm.bme.hu

${ }^{\ddagger}$ University of Bristol, Department of Engineering Mathematics, BS8 1TR, Bristol, UK s.j.hogan@bristol.ac.uk
} 
2. Characteristic matrices. It is well known $[7,18]$ that linear autonomous delay equations admit the construction of a characteristic matrix. Thus, the task of finding the spectra of the infinite dimensional problem is reduced to finding the roots of a nonlinear function, which is just the determinant of the characteristic matrix. Jordan chains [7] of the characteristic matrix at a spectral point can be used to obtain (generalized) eigenvectors of the infinitesimal generator.

For periodic DDEs, there is no such general characteristic matrix, except for special cases. For example, where the delay is an integer multiple of the period length and the partial derivatives of the vector field with respect to the delayed arguments are invertible, Verduyn Lunel [22] constructed a characteristic matrix. This case can also be found in [18], where an equivalence with the monodromy operator is derived (as in the autonomous case) based on the theory presented in [20].

Unfortunately many important practical problems, such as our first test example of interrupted machining (see Sect. 6.1), have noninvertible partial derivatives, so the case in [22] is not applicable. This difficulty can be overcome using Floquet theory [17] and a characteristic matrix can be constructed for integer delays [25]. Although this latter problem does not give equivalence with the monodromy operator, it serves as a starting point of our more general case.

In what follows, we generalize the results of Kaashoek and Verduyn Lunel [20] to periodic delay equations. Let $x$ be a continuous function $x: I \subset \mathbb{R} \rightarrow \mathbb{R}^{n}$ and let $h>0$ be a real number. Define $x_{t} \in C\left([-h, 0], \mathbb{R}^{n}\right)$ by $x_{t}(\theta)=x(t+\theta)$ and consider the following delay-differential equation

$$
x^{\prime}(t)=f\left(t, x_{t}\right),
$$

where $f: \mathbb{R}^{n} \times C\left([-h, 0], \mathbb{R}^{n}\right) \rightarrow \mathbb{R}^{n}$ is of class $C^{N}, N \geq 2$ in the second variable, and the prime denotes time-derivative. Further, assume that $f$ is periodic in the first variable, so $f(t,)=.f(t+\omega,$.$) with \omega \geq h$. Consider a $\omega$-periodic solution $v(t)$ of (2.1). Since $f$ is smooth, we can linearize (2.1) at $v$ in the form

$$
x^{\prime}(t)=\int_{0}^{h} d_{\theta} \eta^{v}(t, \theta) x(t-\theta),
$$

where $\eta^{v}$ is a matrix-valued function that is of bounded variation in $\theta$, and it is associated with the periodic solution $v$. We can represent $\eta$ uniquely if we take the space $N B V$, which consists of functions of bounded variations normalized such that $\eta(\theta)=0$ for $\theta \in(-\infty, 0]$, right continuous on $(0, h)$ and constant on $[h, \infty)$. According to the basic theory of delay equations, (2.2) has a unique (forward) solution if we specify an initial function with $x_{s}=\phi, s \in \mathbb{R}$ fixed. The linearized equation can be written equivalently

$$
x^{\prime}(t)=\int_{0}^{t-s} d_{\theta} \eta^{v}(t, \theta) x(t-\theta)+\int_{t-s}^{\infty} d_{\theta} \eta^{v}(t, \theta) \phi(t-s-\theta),
$$

where the first term includes merely the unknown function, and the second term includes the initial function. Since the initial function is in the equation now, we can solve it uniquely by specifying an initial value $x(s)=\phi(0)$ only.

In what follows, we will consider a bigger space $X=L^{\infty}\left([-\omega, 0], \mathbb{R}^{n}\right)$, where $C\left([-\omega, 0], \mathbb{R}^{n}\right)$ is a closed subspace in $X$. This choice of $X$ guarantees that there is a one-to-one relationship between solutions of (2.2) and the solutions given by our extended monodromy operator (defined below) by maintaining the compatibility with 
the duality framework for delay equations developed by Clement et al. [2, 3] (see also Diekmann et al. [7]). Let us define the operator

$$
(\mathcal{A}(v, s) \varphi)(\theta)=\varphi^{\prime}(\theta)-\int_{0}^{\omega+\theta} d_{\vartheta} \eta^{v}(s+\theta, \vartheta) \varphi(\theta-\vartheta)
$$

with domain of definition $D(\mathcal{A}(v, s))=\operatorname{Lip}\left([-\omega, 0], \mathbb{R}^{n}\right)$ and

$$
(\mathcal{B}(v, s) \varphi)(\theta)=\int_{\omega+\theta}^{\omega} d_{\vartheta} \eta^{v}(s+\theta, \vartheta) \varphi(\omega+\theta-\vartheta) .
$$

on $X$. Note that $\overline{D(\mathcal{A}(v, s))}=C\left([-\omega, 0], \mathbb{R}^{n}\right)$ and $\mathcal{A}(v, s)$ has an $n$ dimensional kernel since, as we mentioned, we can solve $\mathcal{A}(v, s) \varphi=0$ by specifying $\varphi(-\omega)=c$, for any $c \in$ $\mathbb{R}^{n}$. To obtain the monodromy operator, we solve the equation $\mathcal{A}(v, s) \psi=\mathcal{B}(v, s) \varphi$ (which is just a reformulation of (2.3)) with the boundary condition $\psi(-\omega)=\varphi(0)$. Thus the monodromy operator becomes

$$
U(s+\omega, s) \varphi=\psi .
$$

The boundary condition can be eliminated by introducing the (even) bigger space $\hat{X}=\mathbb{R}^{n} \times X$, which includes the initial function $\phi$ and its initial value $\phi(0)$, as well. For ease of notation we use the following boundary value operators

$$
\begin{aligned}
& L: X \rightarrow \mathbb{R}^{n}, L \varphi=\varphi(-\omega), \\
& M: X \rightarrow \mathbb{R}^{n}, M \varphi=\varphi(0) .
\end{aligned}
$$

These definitions make sense, since we consider only functions in the domain of $\mathcal{A}(v, s)$. Let

$$
\hat{\mathcal{A}}(v, s)=\left(\begin{array}{cc}
0 & L \\
0 & \mathcal{A}(v, s)
\end{array}\right)
$$

and

$$
D(\hat{\mathcal{A}}(v, s))=\{(c, \varphi) \in \hat{X} \mid \varphi \in D(\mathcal{A}(v, s)), c=M \varphi\} .
$$

With this definition, it is easy to check that $\hat{\mathcal{A}}(v, s)$ is one-to-one and onto. $\hat{\mathcal{B}}(v, s)$ is defined similarly

$$
\hat{\mathcal{B}}(v, s)=\left(\begin{array}{cc}
I & 0 \\
0 & \mathcal{B}(v, s)
\end{array}\right) .
$$

The extended monodromy operator becomes $\hat{U}(s+\omega, s)=\hat{\mathcal{A}}^{-1}(v, s) \hat{\mathcal{B}}(v, s)$. Note that

$$
\hat{\mathcal{A}}(v, s)\left(\begin{array}{c}
\psi(0) \\
\psi
\end{array}\right)=\hat{\mathcal{B}}(v, s)\left(\begin{array}{c}
\varphi(0) \\
\varphi
\end{array}\right) \Leftrightarrow \psi(-\omega)=\varphi(0), \mathcal{A} \psi=\mathcal{B} \varphi
$$

gives (2.6).

It can easily be proved that the eigenvalues of $\hat{U}(s+\omega, s)$ and $U(s+\omega, s)$ are the same (see [20, Proposition 1.1.]). Therefore we can equivalently analyze the spectrum

$$
\sigma(\hat{U}(s+\omega, s)):=\mathbb{C} \backslash\left\{\lambda \in \mathbb{C} \mid(\lambda I-\hat{U}(s+\omega, s))^{-1} \text { is a bounded linear operator }\right\} .
$$


In case of delay equations, $\hat{U}(s+\omega, s)$ is compact: thus, its spectrum consists of eigenvalues which have a cluster point at 0 . Since we are not interested in 0 , we can define $\mu=1 / \lambda$ and analyze the equivalent problem

$$
\lambda I-\hat{U}(s+\omega, s)=\frac{1}{\mu} \hat{\mathcal{A}}^{-1}(v, s)(\hat{\mathcal{A}}(v, s)-\mu \hat{\mathcal{B}}(v, s))
$$

or, for the sake of simplicity, $\hat{\mathcal{A}}(v, s)-\mu \hat{\mathcal{B}}(v, s)$. In this latter case,

$$
\sigma(\hat{U}(s+\omega, s))=\mathbb{C} \backslash\left\{\frac{1}{\mu} \in \mathbb{C} \mid(\hat{\mathcal{A}}(v, s)-\mu \hat{\mathcal{B}}(v, s))^{-1} \text { is a bounded linear operator }\right\} .
$$

From now on, we suppress the initial time $s$, the periodic solution $v$ or both in the notation of $\mathcal{A}(v, s), \hat{\mathcal{A}}(v, s), \mathcal{B}(v, s)$ and $\hat{\mathcal{B}}(v, s)$.

2.1. Equivalence. Kaashoek and Verduyn Lunel [20] derived the equivalence of unbounded linear operators including infinitesimal generators of evolutionary systems and a characteristic matrix $\Delta(z)$, with some spectral parameter $z$ under quite general assumptions. Here, we show how general periodic DDEs can fit into their framework, and extend the characteristic matrix constructions for equations with integer delays in [18, Section 8.3].

In accordance with the previous Section, we infer that operator $\mathcal{A}$ has the following properties

1. $\mathcal{N}:=\operatorname{ker}(\mathcal{A})$ is finite dimensional, $\operatorname{dim}(\mathcal{N})=n$ and $\mathcal{N} \neq\{0\}$

2. the operator $\mathcal{A}$ has a restriction $\mathcal{A}_{0}: X \rightarrow X$ such that

(a) $D(\mathcal{A})=\mathcal{N} \oplus D\left(\mathcal{A}_{0}\right)$,

(b) $\Omega:=\left\{\mu \in \mathbb{C} \mid\left(\mu \mathcal{B}-\mathcal{A}_{0}\right)^{-1}\right.$ is a bounded operator on $\left.X\right\} \neq \emptyset$.

Also, denote the isomorphism from $\mathbb{R}^{n}$ onto $\mathcal{N}$ by $\jmath$. In what follows, we will use the graph norm $\|x\|_{\hat{\mathcal{A}}}=\|x\|+\|\hat{\mathcal{A}} x\|$ on $D(\hat{\mathcal{A}})$. Using this norm, $\hat{\mathcal{A}}$ becomes bounded and $\hat{X}_{A}:=D(\hat{\mathcal{A}})$ becomes a closed space (see Hille and Phillips [19]).

THEOREM 2.1. If the above conditions hold, we have the equivalence

$$
F(\mu)(\hat{\mathcal{A}}-\mu \hat{\mathcal{B}}) E(\mu)=\left(\begin{array}{cc}
\Delta(\mu) & 0 \\
0 & I_{X}
\end{array}\right), \quad \mu \in \Omega
$$

where $E: \Omega \rightarrow \mathcal{L}\left(\hat{X}, \hat{X}_{A}\right)$ and $F: \Omega \rightarrow \mathcal{L}\left(\hat{X}_{A}, \hat{X}\right)$ are holomorphic bijective operator valued functions and

$$
\Delta(\mu)=(\mu M-L)\left(I-\left(\mu \mathcal{B}-\mathcal{A}_{0}\right)^{-1} \mu \mathcal{B}\right) \jmath \quad \mu \in \Omega .
$$

Furthermore

$$
\begin{aligned}
& E(\mu)\left(\begin{array}{c}
c \\
\varphi
\end{array}\right)=\left(\begin{array}{c}
M\left(Q(\mu) j c+\left(\mu \mathcal{B}-\mathcal{A}_{0}\right)^{-1} \varphi\right) \\
Q(\mu) j c+\left(\mu \mathcal{B}-\mathcal{A}_{0}\right)^{-1} \varphi
\end{array}\right), \\
& E^{-1}(\mu)\left(\begin{array}{c}
M \psi \\
\psi
\end{array}\right)=\left(\begin{array}{c}
j^{-1} Q(0) \psi \\
(\mu \mathcal{B}-\mathcal{A}) \psi
\end{array}\right), \\
& F(\mu)\left(\begin{array}{c}
c \\
\varphi
\end{array}\right)=\left(\begin{array}{c}
c-(\mu M-L)\left(\mu \mathcal{B}-\mathcal{A}_{0}\right)^{-1} \varphi \\
\varphi
\end{array}\right), \\
& F^{-1}(\mu)\left(\begin{array}{c}
c \\
\varphi
\end{array}\right)=\left(\begin{array}{c}
c+(\mu M-L)\left(\mu \mathcal{B}-\mathcal{A}_{0}\right)^{-1} \varphi \\
\varphi
\end{array}\right),
\end{aligned}
$$

where

$$
Q(\mu) \varphi=\varphi-\left(\mu \mathcal{B}-\mathcal{A}_{0}\right)^{-1}(\mu \mathcal{B}-\mathcal{A}) \varphi
$$


Proof. Is similar to the one in [20, Theorem 3.1.], therefore it is omitted.

Remark. The proof is based on the observation that for all $\mu \in \Omega,(\mu \mathcal{B}-\mathcal{A})$ has an $n$ dimensional kernel and that the projection onto $\operatorname{ker}(\mu \mathcal{B}-\mathcal{A})$ is $Q(\mu)$. Since $\operatorname{ker} Q(\mu)$ is independent of $\mu, Q\left(\mu_{1}\right)=Q\left(\mu_{1}\right) Q\left(\mu_{2}\right)$ and therefore $Q(\mu) \jmath$ is an isomorphism from $\mathbb{R}^{n}$ to $\operatorname{ker}(\mu \mathcal{B}-\mathcal{A})$. Applying $(M Q(\mu), Q(\mu))^{T}$ to $\mu \hat{\mathcal{B}}-\hat{\mathcal{A}}$, we find that the first equation is the characteristic matrix and the second equation is identically zero.

We need a characteristic matrices around geometrically simple inverse characteristic multipliers, so we make the following

CONJECTURE 2.2. $\left(\mathcal{A}_{0}-\mu \mathcal{B}\right)$ has a bounded inverse in the following cases:

(i) $\mu^{-1}$ is not a characteristic multiplier;

(ii) $\mu^{-1}$ is a geometrically simple characteristic multiplier of a "generic" periodic orbit.

In continuation the inverse of $\mathcal{A}_{0}-\mu \mathcal{B}$ is numerically computed using LU decomposition, so that the above Conjecture can be checked by monitoring the smallest diagonal element of the $\mathrm{U}$ factor. In our examples in Section 6 this minimal diagonal element always stayed away from zero.

Explanation. First we expand $(\mathcal{A}-\mu \mathcal{B}) \varphi=\psi, L \varphi=c$ into

$$
\dot{\varphi}(\theta)=\int_{0}^{\omega+\theta} \mathrm{d}_{\vartheta} \eta(s+\theta, \vartheta) \varphi(\theta-\vartheta)+\mu \int_{\omega+\theta}^{\omega} \mathrm{d}_{\vartheta} \eta(s+\theta, \vartheta) \varphi(\omega+\theta-\vartheta)-\psi(\theta)
$$

by using (2.4) and (2.5), which contains our problem, when $L \varphi=0$. After integration by parts and changing integration limits, we get that

$$
\begin{gathered}
\dot{\varphi}(\theta)=\eta(s+\theta, \omega+\theta) c+\int_{-\omega}^{\theta} \eta(s+\theta, \theta-\vartheta) \dot{\varphi}(\vartheta) \mathrm{d} \vartheta+ \\
+\mu\left(\eta(s+\theta, \omega) \varphi(\theta)-\eta(s+\theta, \omega+\theta) \varphi(0)+\int_{\theta}^{0} \eta(s+\theta, \omega+\theta-\vartheta) \dot{\varphi}(\vartheta) \mathrm{d} \vartheta\right)-\psi(\theta) .
\end{gathered}
$$

Denote $\phi=\dot{\varphi}$, then the equation becomes

$\phi(\theta)=\int_{-\omega}^{\theta} h_{s, \mu}(\theta, \vartheta) \phi(\vartheta) \mathrm{d} \vartheta+\int_{\theta}^{0} k_{s, \mu}(\theta, \vartheta) \phi(\vartheta) \mathrm{d} \vartheta+(\eta(s+\theta, \omega+\theta)+\eta(s-\omega, \omega)) c-\psi(\theta)$,

where

$$
\begin{gathered}
h_{s, \mu}(\theta, \vartheta)=\eta(s+\theta, \theta-\vartheta)+\mu \eta(s+\theta, \omega)-\mu \eta(s+\theta, \omega+\theta), \\
k_{s, \mu}(\theta, \vartheta)=\mu \eta(s+\theta, \omega+\theta-\vartheta)-\mu \eta(s+\theta, \omega+\theta) .
\end{gathered}
$$

For the sake of simplicity define

$$
(S \phi)(\theta)=\int_{-\omega}^{\theta} h_{s, \mu}(\theta, \vartheta) \phi(\vartheta) \mathrm{d} \vartheta+\int_{\theta}^{0} k_{s, \mu}(\theta, \vartheta) \phi(\vartheta) \mathrm{d} \vartheta
$$

which is a Fredholm operator. Hence $\operatorname{ker}(I-S)$ has the same dimension as $\operatorname{ker}(I-S)^{*}$, which implies that $(I-S)$ is onto if and only if it is one-to-one. From equation (2.9) it is clear that $(\mathcal{A}-\mu \mathcal{B})$ has at least an $n$ dimensional kernel. It is also clear that if 
the kernel has exactly $n$ dimensions then there is a unique $\phi$ satisfying $(2.9)$ for $c=0$, which implies that

$$
\varphi(\theta)=\int_{-\omega}^{\theta} \phi(\vartheta) \mathrm{d} \vartheta
$$

Hence it is enough to prove that $\operatorname{dim} \operatorname{ker}(\mathcal{A}-\mu \mathcal{B})=n$ under our assumptions.

When $\mu$ is not a spectrum point of $(\hat{\mathcal{A}}-\mu \hat{\mathcal{B}})$ we prove that $\left(\mathcal{A}_{0}-\mu \mathcal{B}\right)$ is one-toone by contradiction. Assume that $\operatorname{dim} \operatorname{ker}(\mathcal{A}-\mu \mathcal{B})=m, m>n$. This implies the existence of an isomorphism $p: \mathbb{R}^{m} \rightarrow \operatorname{ker}(\mathcal{A}-\mu \mathcal{B})$. In this case $(L-\mu M) p$ has an at least $m-n$ dimensional kernel, which contradicts our assumption that $(\hat{\mathcal{A}}-\mu \hat{\mathcal{B}})$ is one-to-one and proves that $\operatorname{dim} \operatorname{ker}(\mathcal{A}-\mu \mathcal{B})=n$.

At a geometrically simple inverse characteristic multiplier $\mu$, by definition, $(\hat{\mathcal{A}}-$ $\mu \hat{\mathcal{B}})$ has a one dimensional kernel spanned by $\left(M \varphi_{0}, \varphi_{0}\right)$. In this case we either have $\operatorname{dim} \operatorname{ker}(\mathcal{A}-\mu \mathcal{B})=n$ or $n+1$ by a similar argument as above. We have to prove that the latter case does not occur generically. If $\operatorname{dim} \operatorname{ker}(\mathcal{A}-\mu \mathcal{B})=n+1$ we then have the isomorphism $p: \mathbb{R}^{n+1} \rightarrow \operatorname{ker}(\mathcal{A}-\mu \mathcal{B})$ so that $\operatorname{dim} \operatorname{ker}(L-\mu M) p=1$. However this is not generic, because for any operator, say $F: X \rightarrow \mathbb{R}^{n}$, $\operatorname{dim} \operatorname{ker} F p \geqslant 1$. In order to fix this we need $\operatorname{dim} \operatorname{ker}(\mathcal{A}-\mu \mathcal{B})=n$ and this must hold for almost every periodic orbit with a geometrically simple singularity of a parameter dependent system within a parameter interval. If it is true, the same reasoning applies as above, and we infer that $\left(\mathcal{A}_{0}-\mu \mathcal{B}\right)$ is one-to-one and onto.

In numerical applications, computing (2.8) is not efficient. Instead, we use the isomorphism $R(\mu): \mathbb{R}^{n} \rightarrow \operatorname{ker}(\mu \mathcal{B}-\mathcal{A})$,

$$
R(\mu) c=\left(\begin{array}{ll}
0 & I
\end{array}\right)\left(\begin{array}{cc}
0 & -L \\
0 & \mu \mathcal{B}-\mathcal{A}
\end{array}\right)^{-1}\left(\begin{array}{l}
c \\
0
\end{array}\right),
$$

which is well defined if

$$
D\left(\left(\begin{array}{cc}
0 & -L \\
0 & \mu \mathcal{B}-\mathcal{A}
\end{array}\right)\right)=D(\hat{\mathcal{A}})
$$

in light of Conjecture 2.2. In this case, the equivalent characteristic matrix becomes

$$
\tilde{\Delta}(\mu)=(\mu M-L) R(\mu) \text {. }
$$

3. Continuation of periodic orbits. Define the following nonlinear operator

$$
G: X \times \mathbb{R}^{p} \rightarrow X, \quad G(\varphi, \lambda)(\theta)=x^{\prime}(\theta)-f\left(\theta, \imath_{\theta}(\varphi), \lambda\right),
$$

where $t \in[-T, 0],\left(\imath_{t}(\varphi)\right)(\theta)=\varphi((t+\theta) \bmod \omega)$. The domain of $G$ is $D(G(., \lambda))=$ $\left\{\varphi \in \operatorname{Lip}\left([-\omega, 0], \mathbb{R}^{n}\right) \mid \varphi(-\omega)=\varphi(0)\right\}$. We can extend this operator in the same way as in the previous section:

$$
\hat{G}((c, \varphi), \lambda)=\left(\begin{array}{c}
L \varphi-c \\
G(\varphi, \lambda)
\end{array}\right), \quad(c, \varphi) \in D(\hat{G}(., \lambda))=D(\hat{\mathcal{A}})
$$

Now we have the nonlinear equation for the periodic solutions of (2.1) in the form

$$
\hat{G}((c, \varphi), \lambda)=\left(\begin{array}{l}
0 \\
0
\end{array}\right)
$$

Let $\left(c_{0}, \varphi_{0}\right)=u_{0}$ be a solution of (3.1) at $\lambda_{0}$. Then the Frechet derivative of (3.1) is $\hat{\mathcal{A}}\left(\varphi_{0}\right)-\hat{\mathcal{B}}\left(\varphi_{0}\right)$. By the equivalence $(2.7)$ we infer that $\hat{\mathcal{A}}\left(\varphi_{0}\right)-\hat{\mathcal{B}}\left(\varphi_{0}\right)$ is one-to-one and onto at a regular solution, when there is no +1 multiplier of the monodromy operator $(\operatorname{det} \Delta(1) \neq 0)$. In this case, the solution $u_{0}$ can be continued according to the Implicit Function Theorem. 
3.1. Pseudo-arclength continuation. In our computations, we use the pseudoarclength method to continue branches beyond limit points [10]. Here, we will use the following bilinear form

$$
\langle,\rangle: \hat{X}_{A} \times \hat{X}_{A} \rightarrow \mathbb{R}, \quad\langle u, v\rangle=(M v)^{*} M u+\frac{1}{\omega} \int_{-\omega}^{0} u^{*}(\theta) v(\theta) d \theta
$$

where the ${ }^{*}$ denotes the (conjugate) transpose. Let us denote a branch of solutions by $(u, \lambda): I \subset \mathbb{R} \rightarrow \hat{X} \times \mathbb{R}$ parametrized with the arclength $\gamma$ with respect to the norm $\|(u, \lambda)\|=\sqrt{\langle u, u\rangle}+|\lambda|$. Consider a solution $\left(u_{0}, \lambda_{0}\right)=(u(0), \lambda(0))$, which we want to continue in $\lambda$. Since $u_{0}$ is a regular solution, we can obtain the tangents $\dot{u}_{0}$, $\dot{\lambda}_{0}$ from the equation

$$
\left(\hat{\mathcal{A}}\left(u_{0}\right)-\hat{\mathcal{B}}\left(u_{0}\right)\right) \dot{u}_{0}+D_{\lambda} \hat{G}\left(u_{0}, \lambda\right) \dot{\lambda}_{0}=0 .
$$

Further, we normalize the tangents such that $\left\|\left(\dot{u}_{0}, \dot{\lambda}_{0}\right)\right\|=1$. With these tangents we predict the next solution point on the branch of solutions $u_{1}^{(0)}=d \gamma \dot{u}_{0}, \lambda_{1}^{(0)}=d \gamma \dot{\lambda}_{0}$, where $d \gamma$ is the step-size. Newton iteration of the correction step proceeds by solving

$$
\begin{aligned}
\left(\begin{array}{cc}
\hat{\mathcal{A}}\left(u_{1}^{(\nu)}\right)-\hat{\mathcal{B}}\left(u_{1}^{(\nu)}\right) & D_{\lambda} \hat{G}\left(u_{1}^{(\nu)}, \lambda_{1}^{(\nu)}\right) \\
\left\langle\dot{u}_{0}, .\right\rangle & \dot{\lambda}_{0}
\end{array}\right)\left(\begin{array}{c}
\Delta u \\
\Delta \lambda
\end{array}\right) \\
\quad=\left(\begin{array}{c}
-\hat{G}\left(u_{1}^{(\nu)}, \lambda_{1}^{(\nu)}\right) \\
d \gamma-\left\langle\dot{u}_{0}, u_{1}^{(\nu)}-u_{0}\right\rangle-\left(\lambda_{1}^{(\nu)}-\lambda_{0}\right) \dot{\lambda}_{0}
\end{array}\right)
\end{aligned}
$$

and updating $u_{1}^{(\nu+1)}=u_{1}^{(\nu)}+\Delta u, \lambda_{1}^{(\nu+1)}=\lambda_{1}^{(\nu)}+\Delta \lambda$. According to bordering theorems (see e.g. [10]), the coefficient operator in (3.2) is also one-to-one and onto, since $\hat{\mathcal{A}}\left(u_{0}\right)-\hat{\mathcal{B}}\left(u_{0}\right)$ is one-to-one and onto and $\dot{\lambda}_{0} \neq 0$ at a regular solution. The same is true in the case of a quadratic fold when $D_{\lambda} \hat{G}\left(u_{0}, \lambda_{0}\right) \notin \mathcal{R}\left(\hat{\mathcal{A}}\left(u_{0}\right)-\hat{\mathcal{B}}\left(u_{0}\right)\right)$.

4. Continuation of bifurcations. In this section we reformulate classical continuation theorems of codimension-one bifurcations for periodic DDEs. Since we have the equivalence with the finite-dimensional characteristic matrix, these classical theorems can be applied almost directly. In order to compute the Jacobians, we will need derivatives of the characteristic matrix with respect to $u, \lambda$ and the spectral parameter $\mu$. For the sake of simplicity (and computational efficiency) we use $\tilde{\Delta}(\mu)$. In general, the derivative of the inverse of an operator valued function $A$ is

$$
\left(D_{x} A^{-1}(x)\right)(y)=-A^{-1}(x)\left(D_{x} A(x)\right)\left(A^{-1}(x), y\right) .
$$

Using this relation

$$
\begin{aligned}
& D_{u} \tilde{\Delta}(\mu) c=-(\mu M-L)\left(\begin{array}{cc}
0 & -L \\
0 & \mu \mathcal{B}-\mathcal{A}
\end{array}\right)^{-1} \times \\
& \times D_{u}\left(\begin{array}{cc}
0 & -L \\
0 & \mu \mathcal{B}(u)-\mathcal{A}(u)
\end{array}\right)\left(\left(\begin{array}{cc}
0 & -L \\
0 & \mu \mathcal{B}-\mathcal{A}
\end{array}\right)^{-1}\left(\begin{array}{l}
c \\
0
\end{array}\right), .\right) \\
& D_{\lambda} \tilde{\Delta}(\mu) c=-(\mu M-L)\left(\begin{array}{cc}
0 & -L \\
0 & \mu \mathcal{B}-\mathcal{A}
\end{array}\right)^{-1} \times \\
& \times D_{\lambda}\left(\begin{array}{cc}
0 & -L \\
0 & \mu \mathcal{B}(u)-\mathcal{A}(u)
\end{array}\right)\left(\begin{array}{cc}
0 & -L \\
0 & \mu \mathcal{B}-\mathcal{A}
\end{array}\right)^{-1}\left(\begin{array}{l}
c \\
0
\end{array}\right)
\end{aligned}
$$


and

$$
\begin{aligned}
D_{\mu} \tilde{\Delta}(\mu) c=M R(\mu) c-(\mu M- & L)\left(\begin{array}{cc}
0 & -L \\
0 & \mu \mathcal{B}-\mathcal{A}
\end{array}\right)^{-1} \times \\
\times & \left(\begin{array}{cc}
0 & 0 \\
0 & \mathcal{B}(u)
\end{array}\right)\left(\begin{array}{cc}
0 & -L \\
0 & \mu \mathcal{B}-\mathcal{A}
\end{array}\right)^{-1}\left(\begin{array}{l}
c \\
0
\end{array}\right) .
\end{aligned}
$$

Note, that in the above formulae, it is always the same operator that is inverted.

4.1. Fold bifurcation. In case of a fold bifurcation there is a +1 eigenvalue of the monodromy operator, which is equivalent to $\operatorname{det} \Delta(1)=0$. This implies that we have unit vectors $p$ and $q,\left(p^{*} p=1, q^{*} q=1\right)$ such that $p^{*} \Delta(1)=0$ and $\Delta(1) q=0$. The kernel of $\hat{\mathcal{A}}-\hat{\mathcal{B}}$ and $(\hat{\mathcal{A}}-\hat{\mathcal{B}})^{*}$ is spanned by $\varphi=E(1)(q, 0)^{T}$ and $\psi=F^{*}(1)(p, 0)^{T}$, respectively. The defining system is (see e.g. Beyn et al. [1])

$$
\begin{aligned}
\hat{G}(u, \lambda) & =0, \\
\tilde{\Delta}(1) q & =0, \\
q_{0}^{*} q-1 & =0 .
\end{aligned}
$$

The Jacobian with respect to $(u, q, \lambda)$ is

$$
\left(\begin{array}{ccc}
\hat{\mathcal{A}}\left(u_{0}\right)-\hat{\mathcal{B}}\left(u_{0}\right) & 0 & D_{\lambda} \hat{G}\left(u_{0}, \lambda_{0}\right) \\
D_{u} \tilde{\Delta}(1) q_{0} & \tilde{\Delta}(1) & D_{\lambda} \tilde{\Delta}(1) q_{0} \\
0 & q_{0}^{*} & 0
\end{array}\right) .
$$

Proposition 4.1. The Jacobian in (4.3) is one-to-one and onto at a quadratic fold.

Proof. Same as for algebraic systems, therefore omitted. (see Doedel et al. [10, Section 2.3.]).

4.2. Period doubling. Similarly, the defining system is

$$
\begin{aligned}
\hat{G}(u, \lambda) & =0, \\
\tilde{\Delta}(-1) q & =0, \\
q_{0}^{*} q-1 & =0 .
\end{aligned}
$$

and the Jacobian with respect to $(u, q, \lambda)$ yields

$$
\left(\begin{array}{ccc}
\hat{\mathcal{A}}\left(u_{0}\right)-\hat{\mathcal{B}}\left(u_{0}\right) & 0 & D_{\lambda} \hat{G}\left(u_{0}, \lambda_{0}\right) \\
D_{u} \tilde{\Delta}(-1) q_{0} & \tilde{\Delta}(-1) & D_{\lambda} \tilde{\Delta}(-1) q_{0} \\
0 & q_{0}^{*} & 0
\end{array}\right) .
$$

4.3. Neimark-Sacker bifurcation. The defining system is

$$
\begin{aligned}
\hat{G}(u, \lambda) & =0, \\
\tilde{\Delta}\left(e^{i \alpha}\right) q & =0, \\
q_{0}^{*} q-1 & =0
\end{aligned}
$$

and its Jacobian with respect to $(u, q, \alpha, \lambda)$ becomes

$$
\left(\begin{array}{cccc}
\hat{\mathcal{A}}\left(u_{0}\right)-\hat{\mathcal{B}}\left(u_{0}\right) & 0 & 0 & D_{\lambda} \hat{G}\left(u_{0}, \lambda_{0}\right) \\
D_{u} \tilde{\Delta}\left(e^{i \alpha_{0}}\right) q_{0} & \tilde{\Delta}\left(e^{i \alpha_{0}}\right) & D_{\alpha} \tilde{\Delta}\left(e^{i \alpha_{0}}\right) q_{0} & D_{\lambda} \tilde{\Delta}\left(e^{i \alpha_{0}}\right) q_{0} \\
0 & q_{0}^{*} & 0 & 0
\end{array}\right)
$$


Here we use complex values in the last two rows of the Jacobian, which can be expanded to reals, and then use the same argument to prove the regularity as in $[10$, Section 2.3.].

4.4. Remark on the autonomous case. The presented method can easily be extended for autonomous equations by adding a phase condition to the defining systems and the period to the list of unknown variables [12]. In the case of a fold bifurcation in autonomous systems, the characteristic multiplier +1 has algebraic multiplicity two, therefore, it is not sufficient to use $\Delta(1) q$, but by the equivalence of Jordan chains [20],

$$
\begin{aligned}
\tilde{\Delta}(1) q_{1} & =0, \\
D_{\mu} \tilde{\Delta}(1) q_{1}+\tilde{\Delta}(1) q_{2} & =0 .
\end{aligned}
$$

The size of this system of equations can be reduced by computing $q_{1}$ and $D_{\mu} \Delta(1) q_{1}$. The eigenfunction $\phi_{1}$ of the trivial multiplier 1 is always the derivative of the solution, which equals the right hand side of the equation $\phi_{1}(\theta)=f\left(\imath_{\theta}\left(u_{0}\right), \lambda\right)$. Further, from the analysis above, we infer that $q_{1}=M \phi_{1}=f\left(\imath_{(0)}\left(u_{0}\right), \lambda\right)$. On the other hand, $D_{\mu} \Delta(1) q_{1}$ can be computed according to (4.2) as

$$
D_{\mu} \tilde{\Delta}(\mu) q_{1}=f\left(i_{(0)}(u), \lambda\right)-M\left(\begin{array}{cc}
0 & -L \\
0 & \mathcal{B}-\mathcal{A}
\end{array}\right)^{-1}\left(\begin{array}{c}
0 \\
\mathcal{B} f\left(i_{(.)}(u), \lambda\right)
\end{array}\right) .
$$

In general, Eqn. (4.6) defines a one dimensional kernel space at a regular solution, which corresponds to $q_{1}$. However, if there is a algebraically double +1 multiplier our system (4.6) has a two dimensional kernel space. To eliminate the trivial kernel we use bordering, which reduces (4.6) to

$$
\left(\begin{array}{cc}
D_{\mu} \tilde{\Delta}(\mu) q_{1} & \tilde{\Delta}(\mu) \\
0 & q_{1}^{*}
\end{array}\right) \tilde{q}_{2}=0
$$

where $\tilde{q}_{2}$ is a new variable, which contains $q_{2}$ from its second element. We remark that in [8], test functionals for folds in ODEs are constructed in a similar way.

5. Implementation. In this section, we summarize the numerical methods used in our continuation software that uses orthogonal collocation [5] to discretize the operators $\mathcal{A}$ and $\mathcal{B}$ of equations with discrete fixed delays. Thus, equation (2.1) can be written as

$$
x^{\prime}(\tilde{t})=\tilde{f}\left(\tilde{t}, x\left(\tilde{t}-\tilde{\tau}_{1}\right), \ldots, x\left(\tilde{t}-\tilde{\tau}_{m}\right)\right),
$$

where $\tilde{f}: \mathbb{T}^{1} \times \mathbb{R}^{n} \times \cdots \times \mathbb{R}^{n} \rightarrow \mathbb{R}^{n}$ and $0 \leq \tilde{\tau}_{1}<\tilde{\tau}_{2}<\cdots<\tilde{\tau}_{m} \leq \omega$. By rescaling the time with $\tilde{t}=\omega t$ and defining $f\left(t, x_{1}, \ldots, x_{m}\right)=\tilde{f}\left(\omega t, x_{1}, \ldots, x_{m}\right)$, our equation becomes

$$
x^{\prime}(t)=\omega f\left(t, x\left(t-\tau_{1}\right), \ldots, x\left(t-\tau_{m}\right)\right) .
$$

Its variational system at $v$ is

$$
x^{\prime}(t)=\sum_{i=1}^{m} D_{x_{i}} f\left(t, v\left(t-\tau_{1}\right), \ldots, v\left(t-\tau_{m}\right)\right) x\left(t-\tau_{i}\right) .
$$


Also, assume that the initial time $s=0$. Let $A_{i}, B_{i}: X \times[-1,0] \rightarrow \mathbb{R}^{n \times n}$ defined by

$$
\begin{aligned}
& A_{i}(v, \theta)=\omega H\left(1+\theta-\tau_{i}\right) D_{x_{i}} f\left(\theta, v\left(\left(\theta-\tau_{1}\right) \bmod 1\right), \ldots, v\left(\left(\theta-\tau_{m}\right) \bmod 1\right)\right), \\
& B_{i}(v, \theta)=\omega H\left(\tau_{i}-1-\theta\right) D_{x_{i}} f\left(\theta, v\left(\left(\theta-\tau_{1}\right) \bmod 1\right), \ldots, v\left(\left(\theta-\tau_{m}\right) \bmod 1\right)\right),
\end{aligned}
$$

where mod maps to $[-1,0]$ and $H$ is the Heaviside function. Then (2.4) and (2.5) become

$$
\begin{gathered}
(\mathcal{A}(v) \varphi)(\theta)=\varphi^{\prime}(\theta)-\sum_{i=1}^{m} A_{i}(\theta) \varphi\left(\theta-\tau_{i}\right) \\
(\mathcal{B}(v) \varphi)(\theta)=\sum_{i=1}^{m} B_{i}(\theta) \varphi\left(1+\theta-\tau_{i}\right)
\end{gathered}
$$

5.1. Collocation. Here, we summarize the main steps of the method, its detailed discussion can be found in [12]. We approximate the solution $\varphi$ on $\mathfrak{n}$ intervals by degree $\mathfrak{m}$ polynomials. The method requires the space $X$ to be $C^{\mathfrak{m}}\left([-1,0], \mathbb{R}^{n}\right)$ and consequently $D(\mathcal{A}(v))=C^{\mathfrak{m}+1}\left([-1,0], \mathbb{R}^{n}\right)$ and $f$ also $C^{\mathfrak{m}}$-smooth in $t$. The mesh is represented by the intervals $\left[\theta_{i}, \theta_{i+1}\right], i=0, \ldots, \mathfrak{n}-1$ with $-1=\theta_{0}<\theta_{1}<\cdots<\theta_{\mathfrak{n}}=$ 0 . Also denote the length of an interval by $h_{i}:=\theta_{i+1}-\theta_{i}$. The approximate solution can be written on the $i$-th subinterval as

$$
\tilde{\varphi}(\theta)=\sum_{j=0}^{\mathfrak{m}} \varphi\left(\theta_{i+\frac{j}{\mathfrak{m}}}\right) P_{i, j}(\theta)
$$

where

$$
P_{i, j}(\theta)=\prod_{r=0, r \neq j}^{\mathfrak{m}} \frac{\theta-\theta_{i+\frac{r}{\mathfrak{m}}}}{\theta_{i+\frac{j}{\mathfrak{m}}}-\theta_{i+\frac{r}{\mathfrak{m}}}}
$$

and $\theta_{i+\frac{j}{\mathfrak{m}}}:=\theta_{i}+j \frac{h_{i}}{\mathfrak{m}}$. Let $c_{i}, i=1, \ldots \mathfrak{m}-1$ be the roots of the degree $\mathfrak{m}$ Legendre polynomial scaled from $[-1,1]$ to $[0,1]$. Then define the collocation points by $c_{j, i}=$ $\theta_{j}+h_{j} c_{i}$. The discretized version of operator $\mathcal{A}$ becomes

$$
\left(\mathcal{A}_{d}(v) \tilde{\varphi}\right)\left(c_{j, i}\right)=\tilde{\varphi}^{\prime}\left(c_{j, i}\right)-\sum_{i=1}^{m} A_{i}\left(c_{j, i}\right) \tilde{\varphi}\left(c_{j, i}-\tau_{i}\right) .
$$

Operator $\mathcal{B}$ is discretized similarly. Note that (5.1) is an operator from $\mathbb{R}^{(\mathfrak{m} \times \mathfrak{n}-1) \times n}$ to $\mathbb{R}^{\mathfrak{m} \times \mathfrak{n} \times n}$, so it has (at least) an $n$ dimesional kernel just as its abstract counterpart (2.4) has. The discretized version of $\hat{\mathcal{A}}$ acting on $(M \varphi, \varphi)$ can be represented by a square matrix by placing the $-L \varphi$ vector into the first $n$ rows, which is of full rank at a regular solution $v$ according to [11, Theorem 3.1] when $|h|=\max _{i=0}^{n-1} h_{i}$ is sufficiently small. Moreover, if we choose the space $X=C^{\mathfrak{m}}\left([-1,0], \mathbb{R}^{n}\right)$ as described above then there exists a $\delta>0$ and $C>0$ such that the solutions of $\hat{\mathcal{A}} \psi=\hat{\mathcal{B}} \varphi$ and $\hat{\mathcal{A}}_{d} \tilde{\psi}=\hat{\mathcal{B}}_{d} \tilde{\varphi}$ satisfy

$$
\max _{\theta \in[-1,0]}|\psi(\theta)-\tilde{\psi}(\theta)| \leq C|h|^{\mathfrak{m}}
$$

if $|h| \in(0, \delta]$ (see Engelborghs and Doedel [11, Theorem 3.3]). Although superconvergence is lost for delay equations, some improvement can be achieved over (5.2) by using modified collocation schemes (see [11, Section 4] and [12]). 
5.2. Solving bordered linear systems. Equations (3.2), (4.3), (4.4) and (4.5) contain the operator $\hat{\mathcal{A}}-\hat{\mathcal{B}}$, functionals like $\left\langle\dot{u}_{0},.\right\rangle$ on $\hat{X}$, vectors like $D_{\lambda} \hat{G}\left(u_{0}, \lambda_{0}\right)$ in $\hat{X}$, and also the finite dimensional characteristic matrix. From a computational point of view, it is better to factorize the discretized counterparts of these components separately, rather than copying them into one big matrix. Usually, the discretized infinite dimensional part has a special sparsity structure, which can be exploited in computations. Other reasons for bordering methods might be the reuse of code and avoiding the frequent copying of large data structures. In our code, we used methods developed by Govaerts $[14,15]$ and Govaerts and Pryce [16]. These methods have the advantage over classical bordering methods $[10,1]$ that they do not require the calculation of the kernel of singular matrices for the computation and can be used with arbitrary stable solvers. This feature makes it possible to use the same routine for sparse (e.g., $\hat{\mathcal{A}}_{d}-\hat{\mathcal{B}}_{d}$ with discrete delays) and dense (e.g., $\hat{\mathcal{A}}_{d}-\hat{\mathcal{B}}_{d}$ with distributed delays) matrices, leaving space for future improvements.

The method of Mixed Block Elimination (BEM) [14] is used in factorizing the discretized counterpart of the Jacobian in Eqn. (3.2). For solving equations of the form

$$
\left(\begin{array}{ll}
A & b \\
c^{*} & d
\end{array}\right)\left(\begin{array}{l}
x \\
y
\end{array}\right)=\left(\begin{array}{l}
\alpha \\
\beta
\end{array}\right),
$$

the method requires a solution with $A$ and one with $A^{*}$. Note, that for this solution $A$ is factorized only once. For the bigger problem

$$
\left(\begin{array}{ccc}
A & 0 & b \\
C & D & e \\
f^{*} & g^{*} & h
\end{array}\right)\left(\begin{array}{l}
x \\
y \\
z
\end{array}\right)=\left(\begin{array}{c}
\alpha \\
\beta \\
\delta
\end{array}\right),
$$

which appears in Eqns. (4.3) and (4.4), we apply the Generalized Mixed Block Elimination (GMBE) [14] technique, which requires one solution with $D^{T}$ and two BEM steps with $A$ and $D$, respectively. In case of systems augmented with wider borders, BEMW [14] is used, which is the iterative form of the recursive version of BEM. The wider borders occur in Eqns. (4.5), (4.3), and also in (4.4) when it is further augmented with derivatives and tangent vectors in the two- parameter continuation context similarly to Eqn. (3.2). These kinds of equations can be solved using GMBE but BEM replaced with BEMW.

Factorization of sparse matrices was carried out using UMFPACK ${ }^{1}$, which implements the Unsymmetric Multifrontal method of direct LU factorization (see Davies [4]). For (nearly) optimal performance we construct the matrices $\hat{\mathcal{A}}_{d}-\hat{\mathcal{B}}_{d}$ directly in compressed row form instead of the default compressed column form. After factorization, we interchange the solution methods for the matrix and its transpose to get the correct results. For dense matrices, we use the standard LAPACK routine GESVX.

6. Examples. Our first example is a forced system which models an interrupted machining process. In this example, when considering period-one solutions, the delay equals the period. However, it is not the case of the period-two solutions where it has two delays, the smallest being half the period length, so the characteristic matrix definition of Lunel [22] is not applicable and we need our general construction. The second example is a traffic model, which demonstrates that our algorithm can handle arbitrary (varying) delays. This second example, being autonomous, also demonstrates the fold continuation in our remark in Section 4.4.

\footnotetext{
${ }^{1}$ We remark that this package is incorporated into MATLAB as well.
} 


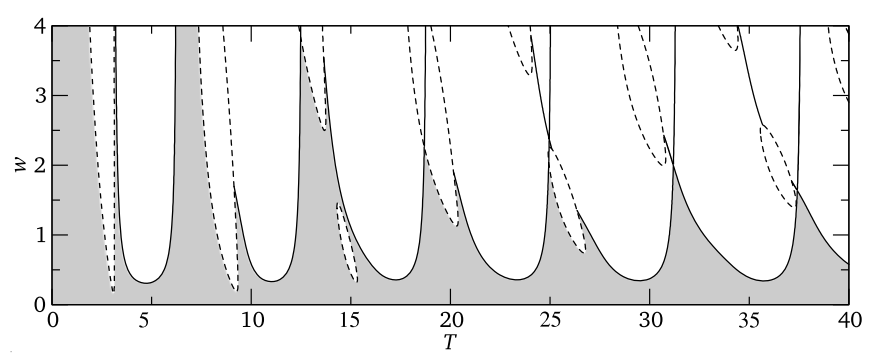

FIG. 6.1. Stability chart for the interrupted machining model showing period doubling boundaries (dashed lines) and Neimark-Sacker boundaries (continuous lines). In the gray region the $T$ periodic solution is stable. $(\zeta=0.015)$

6.1. Interrupted machining. Here, we analyse bifurcations of period-two orbits in an interrupted machining model. To this end, we consider an extended version of the equation of motion in [25] in the form

$$
\begin{array}{r}
x^{\prime \prime}(t)+2 \zeta x^{\prime}(t)+x(t)=w g(t) H(1+x(t-2 T)-x(t-T)) F_{c}(1+x(t-T)-x(t)) \\
+w g(t) H(1+x(t-T)-x(t-2 T)) F_{c}(2+x(t-2 T)-x(t)), \quad(6.1)
\end{array}
$$

where $H$ is the Heaviside function,

$$
g(t)=\left\{\begin{array}{ll}
0 & \text { if } \exists k: k T \leq t<(k+1-\rho) T \\
1 & \text { if } \exists k:(k-\rho) T \leq t<k T
\end{array}, \quad k \in \mathbb{Z}\right.
$$

and

$$
F_{c}(x)=H(x)\left(\frac{120}{59} x-\frac{48}{59} x^{2}+\frac{35}{177} x^{3}\right) .
$$

This cutting force function $F_{c}$ is a polynomial approximation of the common threequarter rule $F_{c}(x) \approx 4 / 3 x^{3 / 4}$ (see [27]). Also, $\zeta=0.015$ is the relative damping, $\rho=0.1$ is the ratio between the period length $T$ and the time spent with cutting. Equation (6.1) has a unique $T$-periodic solution, which is described by the ODE

$$
x^{\prime \prime}(t)+2 \zeta x^{\prime}(t)+x(t)=w g(t) F_{c}(1) .
$$

In a quite large neighborhood of this periodic solution, (6.1) simplifies to

$$
x^{\prime \prime}(t)+2 \zeta x^{\prime}(t)+x(t)=w g(t) F_{c}(1+x(t-T)-x(t)),
$$

while the second line of (6.1) is turned on if the tool did not cut the workpiece in the previous period. Its variational equation around the $T$-periodic solution is

$$
x^{\prime \prime}(t)+2 \zeta x^{\prime}(t)+x(t)=w g(t)(x(t-T)-x(t)) .
$$

The stability of the above equation was analyzed analytically in [25] in great detail. The stability chart is shown in Fig. 6.1. We analyze the lowest lens at $T \approx 15$. The bifurcation diagram at $T=14.75$ can be seen in Fig. 6.2. The first period doubling bifurcation at $w \approx 0.6629$ is subcritical. The arising unstable period-two orbit folds back and then loses its stability through a torus bifurcation. The other period doubling bifurcation is supercritical. On the stable branch there is a torus bifurcation window. This branch folds back, too and becomes unstable. 


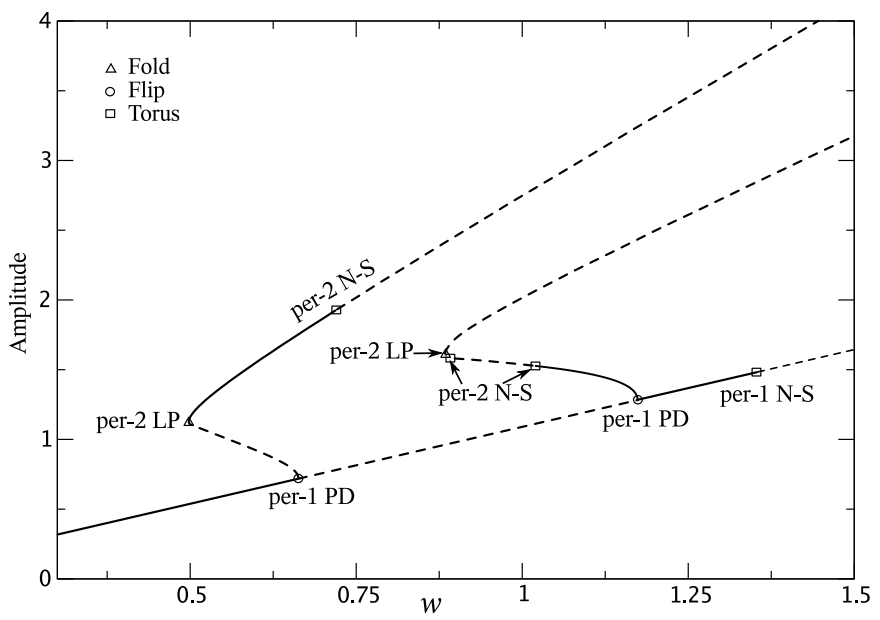

FIG. 6.2. This bifurcation diagram shows the period-two orbits emanating from the period-one solution at the stability boundary in the vertical cross-section of a lens at $T=14.75$ in Fig. 6.1. Continuous lines denote stable solutions while dashed lines correspond to unstable solutions.

The bifurcations in Fig. 6.2 were continued in two parameters using our method. First, the stability boundaries of (6.2) are obtained, which coincide with the semianalytical results of [25]. The period $T$ orbits are also checked analytically. In order to avoid difficulties arising from the non-smoothness of (6.1), we substitute the Heaviside function $H(x)$ by $(1+\tanh (C x)) / 2$ with $C=30$. Choosing bigger values for $C$ is not reasonable because it only improves the results within the line thickness but makes the computation more difficult due to derivatives tending to infinity. The period-two orbits arising at the lens in the space of two parameters extended with the solution amplitude form a highly distorted tube-like object. Figure 6.3 shows the computed bifurcation curves. The lower continuous curve corresponds to the fold bifurcation of orbits arising at the lower part of the lens. The other continuous curve shows the fold of the upper solution branch in Fig. 6.2. It can be seen that there are two cusp points on the bifurcation curve, which in addition, connects to the period doubling boundary of period-one solutions separating sub- and supercritical period doubling bifurcations. The dash-dot lens near to the upper fold curve is an unstable island on the supercritically bifurcated family of period-two solutions, while the other dash-dot line corresponds to the stability loss of motion on the other branch of orbits.

6.2. Traffic model with driver reaction time. Here, we focus on the fold bifurcations of periodic orbits in the autonomous traffic model described in Orosz et al. [23]. We consider $N_{c}$ cars on a circular track of length $L$, so that the distances between the cars $\left(h_{i}\right)$ add up to $L$

$$
\sum_{i} h_{i}(t)=L
$$

and these distances vary with the speed difference between two adjacent cars

$$
\dot{h}_{i}(t)=v_{i+1}(t)-v_{i}(t)
$$

where $v_{i}$ denotes the speed of the $i$ th car. The drivers increase or decrease their speed according to their current speed and the headway before them. However, they can 


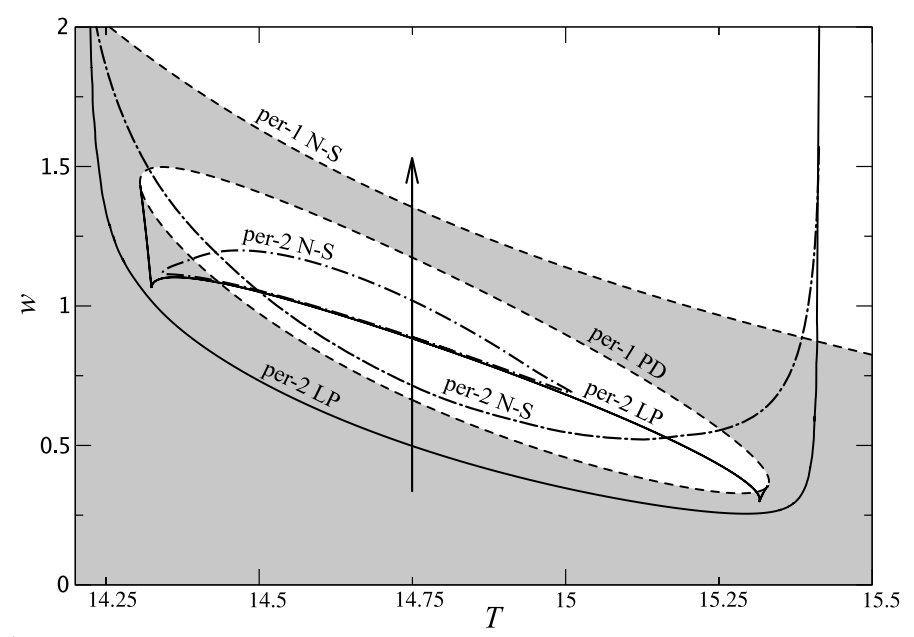

FIG. 6.3. Bifurcations of period-two orbits. Dash lines correspond to the stability of period-two solutions (see Fig. 6.1). Continuous lines are fold bifurcation curves, dash-dot lines are NeimarkSacker stability curves of period-two solutions.

not react immediately, so a delayed value of the headway is considered in the model:

$$
\dot{v}_{i}(t)=\beta\left[V\left(h_{i}(t-\tau)\right)-v_{i}(t)\right],
$$

where $\beta$ is the sensitivity of the drivers and $V$ is the so-called optimal velocity function defined as

$$
V(h)=\left\{\begin{array}{cc}
0 & 0 \leq h \leq 1, \\
v^{0} \frac{(h-1)^{3}}{1+(h-1)^{3}} & h>1
\end{array} .\right.
$$

The steady state solution of the model corresponds to equal headways and equal car velocities, that is,

$$
h_{i}^{*}=L / N, \quad v_{i}^{*}=V\left(h_{i}^{*}\right),
$$

where $N_{c}$ is the number of cars on the track.

From now on we focus on the case $N_{c}=3$, thus our equations become

$$
\begin{aligned}
& \dot{h}_{1}(t)=v_{2}(t)-v_{1}(t), \\
& \dot{h}_{2}(t)=v_{3}(t)-v_{2}(t), \\
& \dot{v}_{1}(t)=\beta\left[V\left(h_{1}(t-\tau)\right)-v_{1}(t)\right], \\
& \dot{v}_{2}(t)=\beta\left[V\left(h_{2}(t-\tau)\right)-v_{2}(t)\right], \\
& \dot{v}_{3}(t)=\beta\left[V\left(L-h_{1}(t-\tau)-h_{2}(t-\tau)\right)-v_{3}(t)\right],
\end{aligned}
$$

using Eqn. 6.3. The fixed points can undergo a Hopf bifurcation. Although it is not computationally efficient, we computed the Hopf bifurcation curve using our method, considering the fixed point as a periodic solution (see Fig. 6.5). After computing the critical eigenvectors of the characteristic matrix we constructed a linearly approximate periodic solution around the fixed point

$$
\begin{aligned}
& h_{i}(t)=\epsilon \operatorname{Re}\left(\mathrm{e}^{i \alpha t} h_{i}^{q}\right), \\
& v_{i}(t)=\epsilon \operatorname{Re}\left(\mathrm{e}^{i \alpha t} v_{i}^{q}\right),
\end{aligned}
$$



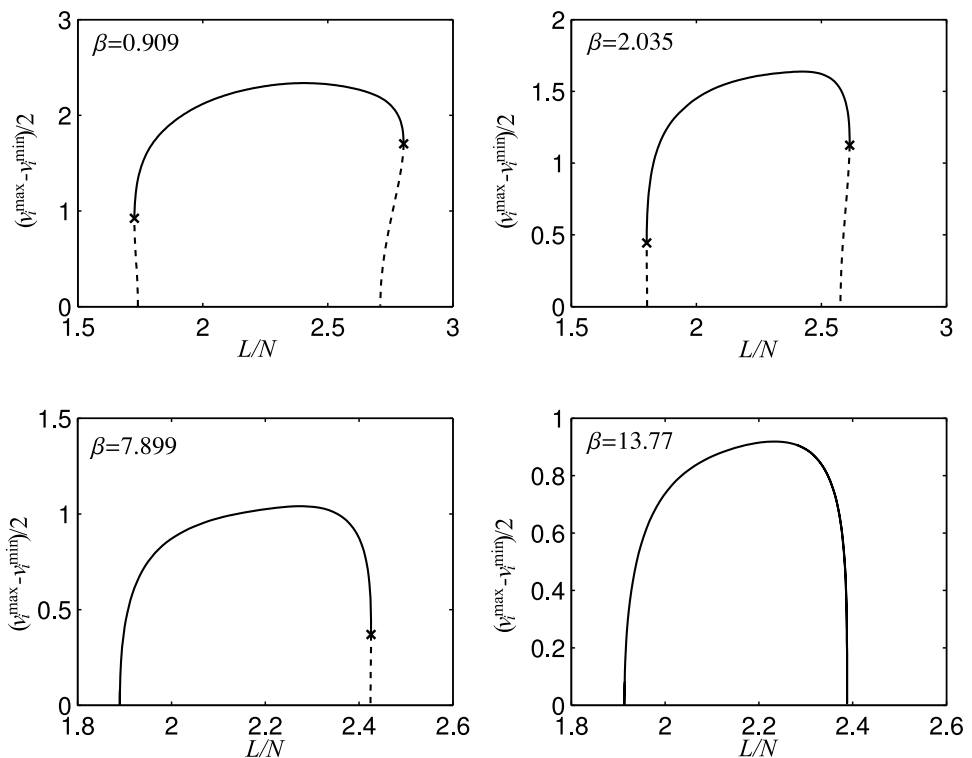

FIG. 6.4. Branches of periodic orbits in the traffic model. Dashed curves represent unstable solutions, while continuous curves denote stable solutions. The parameters are $v^{0}=0.8$ and $\tau=1.0$.

where $\epsilon$ is a sufficiently small real number, $\alpha$ is in the argument of the critical characteristic multiplier (see Eqn. 4.5) and $h_{i}^{q}, v_{i}^{q}$ are the respective components of the kernel vector $q$ of $\Delta\left(\mathrm{e}^{i \alpha}\right)$. The computed branches of periodic solutions for different values of $\beta$ can be seen in Fig. 6.4. These periodic orbits undergo fold bifurcations, whose locations can be continued in two parameters using our method. These fold curves are represented by continuous lines in Fig. 6.5, while the Hopf bifurcation of the fixed point is denoted by the dashed lines. It can be seen that the two type of bifurcations meet in a degenerate Hopf $(\mathrm{DH})$ point, where the monodromy operator has triple +1 eigenvalues including the trivial one.

7. Discussion. In our presentation the characteristic matrix definition was given for equations with delays smaller than or equal to the period. However, our definition can be easily extended to any bounded delays such as the special case of integer delays in [22]. This extended algorithm is already implemented in the software PDDE-CONT [24].

Although the full equivalence of a characteristic matrix and the monodromy operator at spectral points remains to be proven, the correctness of our conjecture can be checked numerically with a minimal effort. For discrete delays $\tau_{i}$ that are rationally related to the period, e.g., $\tau_{i}=r_{i} / p, r_{i}, p \in \mathbb{N}$, one can construct an $n \times p$ dimensional characteristic matrix that is equivalent to the monodromy operator, since the operator in Conjecture 2.2 can be inverted by solving ordinary differential equations. Hence for discrete delays that are sufficiently close to rational delays a characteristic matrix can be constructed, because the resolvent set of an operator is open and the operator is norm continuous with respect to the delays.

The equivalence with the monodromy operator also gives the opportunity to compute center manifolds and normal forms for periodic equations in special cases where the characteristic matrix is analytically available as it was demonstrated in [26] 


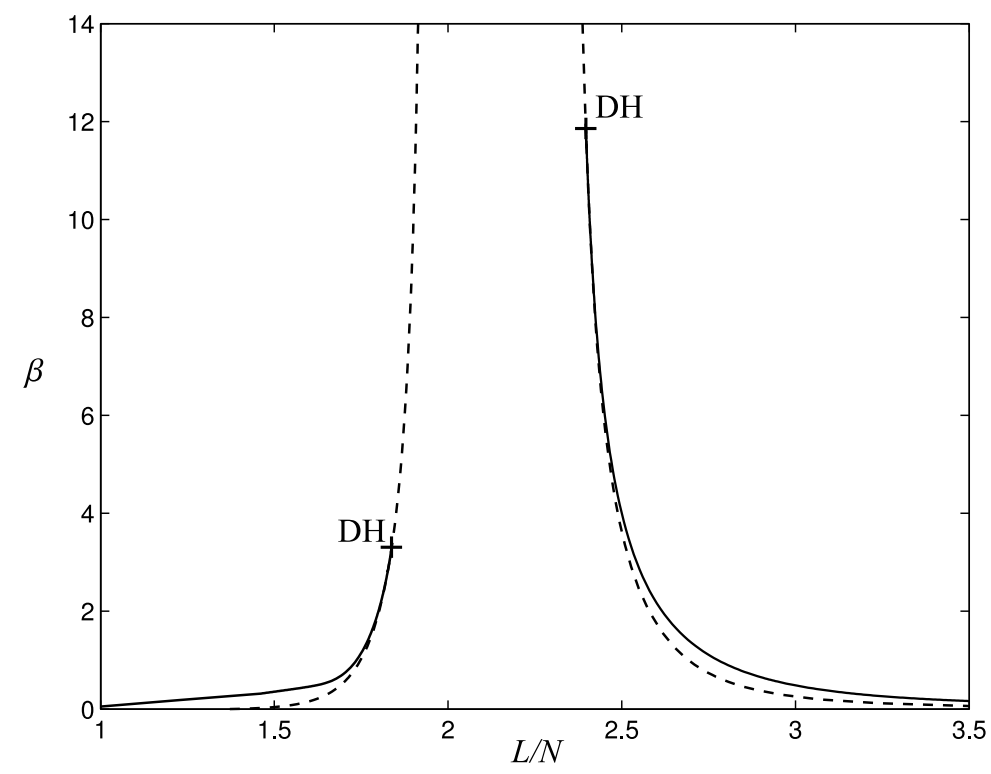

FIG. 6.5. Stability chart. Between the two dashed curves denoting Hopf bifurcations, the fixed point is unstable. The arising periodic orbits fold back along the continuous curve. The fold curves meet the Hopf bifurcation curve in the degenerate Hopf points denoted by ' + ' signs. $\left(v^{0}=0.8\right.$, $\tau=1.0)$

8. Conclusions. Periodic delay-differential equations frequently arise in applications that describe phenomena in engineering, biology, etc. In this paper we have constructed a characteristic matrix for general periodic DDEs in order to use it in the numerical continuation of bifurcations. We also presented the equivalence of the characteristic matrix with the monodromy operator, which makes regularity proofs of defining systems of equations similar to the proofs in the case of finite dimensional algebraic systems. We have implemented the algorithm in software written in $\mathrm{C}++$ [24], which uses collocation to discretize the infinite dimensional operators. Bordering techniques were also used in the solution of linear systems extended with the characteristic matrix, some derivatives, and tangent vectors. Finally, two examples, an interrupted machining model and a traffic model was analyzed.

9. Acknowledgments. The authors thank Bernd Krauskopf, Kirk Green, Gábor Orosz and Barnabás Garay for helpful discussion. During the preparation of this work the first author was supported by the Hungarian Eötvös scholarship and the Fulbright Scholarship. The second author was supported by the Hungarian National Science Foundation under grant no. OTKA T043368.

\section{REFERENCES}

[1] W. J. Beyn, A. Champneys, E. J. Doedel, W. Govaerts, B. Sandstede, And Y. A. KuZnetsov, Numerical continuation and computation of normal forms, in Handbook of Dynamical Systems, B. Fiedler, ed., Elsevier, 2002, pp. 149-219.

[2] P. Clement, O. Diekmann, M. Gyllenberg, H. J. A. M. Heimans, and H. R. Thieme, Perturbation theory for dual semigroups I. The sun-reflexive case, Math. Ann., 277 (1987), pp. 709-725.

[3] - Perturbation theory for dual semigroups II. Time-dependent perturbations in the sunreflexive case, Proc. Roy. Soc. Edinburgh Sect. A, 109A (1988), pp. 145-172. 
[4] T. A. Davies, UMFPACK Version 4.1 User Guide, Tech. Rep., Department of Computer and Information Science and Engineering, University of Florida, Gainesville, FL, USA, 2003. http://www.cise.ufl.edu/research/sparse/umf pack/.

[5] C. De Boor And B. Swartz, Collocation at gaussian points, SIAM J. Numer. Anal., 10 (1973), pp. 582-606.

[6] A. Dhooge, W. Govaerts, and Y. Kuznetsov, matcont: A matlab package for numerical bifurcation analysis of ODEs, ACM Trans. Math. Software, 29 (2003), pp. 141-164.

[7] O. Diekmann, S. A. van Gils, S. M. Verduyn Lunel, and H. O. Walther, Delay equations, Springer-Verlag, New-York, 1995.

[8] E. Doedel, W. Govaerts, And Y. Kuznetsov, Computation of periodic solution bifurcations in odes using bordered system, SIAM J. Numer. Anal., 41 (2003), pp. 401-435.

[9] E. J. Doedel, A. R. Champneys, T. F. Fairgrieve, Y. A. Kuznetsov, B. Sandstede, and X.-J. WANG, AUTO97: Continuation and bifurcation software for ordinary differential equations, Tech. Rep., Department of Computer Science, Concordia University, Montreal, Canada, 1997. (Available by FTP from ftp.cs.concordia.ca in directory pub/doedel/auto).

[10] E. J. Doedel, H. B. Keller, J. P. Kernévez, Numerical analysis and control of bifurcation Problems, Part I : Bifurcation in finite dimensions, Int. J. Bifurcation and Chaos, 1 (1991), pp. 493-520.

[11] K. Engelborghs And E. J. Doedel, Stability of piecewise polynomial collocation for computing periodic solutions of delay differential equations, Numer. Math., 91 (2002), pp. 627-648.

[12] K. Engelborghs, T. Luzyanina, K. J. in 'T Hout, and D. Roose, Collocation methods for the computation of periodic solutions of delay differential equations, SIAM J. Sci. Comput., 22 (2000), pp. 1593-1609.

[13] K. Engelborghs, T. Luzyanina, and D. Roose, Numerical bifurcation analysis of delay differential equations using DDE-BIFTOOL, ACM Trans. Math. Software, 28 (2002), pp. 121.

[14] W. GovaERTs, Bordered augmented linear systems in numerical continuation and bifurcation, Numer. Math., 58 (1990), pp. 353-368.

[15] - Solution of bordered singular systems in numerical continuation and bifurcation, J. Comput. Appl. Math., 50 (1994), pp. 339-347.

[16] W. Govaerts And J. D. Pryce, Mixed block elimination for linear systems with wider borders, IMA J. of Numer. Anal., 13 (1993), pp. 161-180.

[17] J. K. Hale, Theory of functional differential equations, Springer-Verlag, New-York, 1977.

[18] J. K. Hale ANd S. M. Verduyn Lunel, Introduction to functional differential equations, Springer-Verlag, New-York, 1993.

[19] E. Hille AND R. S. Phillips, Functional analysis and semigroups, American Mathematical Society, 190 Hope Street, Providence, R.I., 1957.

[20] M. A. KaAshoek and S. M. V. Lunel, Characteristic matrices and spectral properties of evolutionary systems, Trans. Amer. Math. Soc., 334 (1992), pp. 479-517.

[21] Y. A. KUZNETSOV, CONTENT - integrated environment for analysis of dynamical systems. Tutorial, Tech. Rep., Ecole Normale Superieure de Lyon, 1998. Rapport de Recherche UPMA-98-224.

[22] S. M. VerduYn Lunel, Small solutions and completeness for linear functional differential equations, in Oscillations and Dynamics in Delay Equations, vol. Contemporary Mathematics 129, Amer. Math. Soc., 1992, pp. 127-152.

[23] G. Orosz, R. E. Wilson, and B. Krauskopf, Global bifurcation investigation of an optimal velocity traffic model with driver reaction time, Phys. Rev. E, 70 (2004), pp. 026207 1-10.

[24] R. Szalai, PDDE-CONT: A continuation and bifurcation software for delay-differential equations, Tech. Rep., Budapest University of Technology and Economics, Budapest, Hungary, 2005. http://www.mm.bme.hu/ szalai/pdde/.

[25] R. Szalai and G. StépÁn, Stability boundaries of high-speed milling corresponding to period doubling are essentially closed curves, in Proceedings of IMECE2003 ASME International Mechanical Engineering Congress and R\&D Expo, 2003, pp. 1-6. Paper number DETC03/VIB-48572 (CD-ROM).

[26] R. Szalai And G. StéPÁN, Period doubling bifurcation and center manifold reduction in a time-periodic and time-delayed model of machining, preprint, 2006.

[27] J. Tlusty, Manufacturing process and equipment, Prentice Hall, New Jersey, 2000. 